\title{
On the Performance of Band-Limited Asynchronous DS-CDMA over Nakagami- $m$ Channels
}

\author{
Hua Wei, Student Member, IEEE, Lie-Liang Yang, Senior Member, IEEE, and Lajos Hanzo, Fellow, IEEE
}

\begin{abstract}
In this paper we investigated the BER performance of DS-CDMA using various chip-waveforms, which include three time-limited chip-waveforms and two band-limited chipwaveforms. Closed-form formulae were derived for evaluating the achievable bit-error rate performance with the aid of the standard Gaussian approximation, when communicating over a Nakagami- $m$ channel. The time-limited waveforms impose a low implementational complexity, since they maybe oversampled and read from a look-up table. However, they are outperformed by the frequency-domain raised-cosine waveform as well as the optimum waveform specifically designed by Cho and Lehnert for achieving the lowest possible bit error rate.
\end{abstract}

Index Terms-Code-division multiple-access, chip waveform, Gaussian approximation, Nakagami, band-limited, normalized bandwidth.

\section{INTRODUCTION}

$\mathbf{T}$ HE SIGNALLING pulse design or waveform design plays an important role in determining the properties of digital communication systems. In [1], Amoroso introduced various definitions of the bandwidth of digital signalling schemes. In the context of CDMA based communications [2], numerous valuable studies have been conducted [3]-[7] for the sake of finding various attractive chip waveforms. In [4], the authors investigated the impact of the signalling pulse shape for several time-limited waveforms, such as rectangular, half-sine and raised cosine chip-waveforms. More specifically, the different chip-waveform designs are expected to incur different amounts of multiple access interference (MAI), while requiring different signalling bandwidths. However, the authors of [4] did not consider the bandwidth requirement of the different chip-waveform, although the different chipwaveforms occupy different bandwidths. Hence in this paper we will investigate the BER performance of these time-limited chip-waveforms in the context of a DS-CDMA system, when they occupy the same bandwidth.

Cho and Lehnert [5] investigated the performance of several band-limited chip-waveforms in the context of DS-CDMA systems, when communicating over a non-dispersive AWGN channel. For example, a time-domain chip-waveform associated with a raised cosine spectrum was studied in [5], [8].

Manuscript received June 18, 2004; revised May 10, 2005 and September 1, 2005; accepted September 1, 2005. The associate editor coordinating the review of this letter and approving it for publication was J. Garcia-Frias. The work reported in this paper has formed part of the Wireless Enablers Work Area of the Core 2 Research Programme of the Virtual Centre of Excellence in Mobile \& Personal Communications, Mobile VCE, www.mobilevce.com, whose funding support, including that of EPSRC, is gratefully acknowledged. Fully detailed technical reports on this research are available to Industrial Members of Mobile VCE.

The authors are with the School of Electronics and Computer Science, Univ. of Southampton, SO17 1BJ, UK (e-mail: 1ly@ecs.soton.ac.uk; lh@ecs.soton.ac.uk).

Digital Object Identifier 10.1109/TWC.2006.04423
More specifically, the authors of [5] defined the so-called normalized interference factor for the sake of characterizing the interference-related effects of a specific chip-pulse shape and hence for analyzing the achievable performance of band-limited waveform based DS-CDMA systems, as it will be discussed in detail throughout this paper. The novel contribution of [9] was that the authors provided a precise error-rate analysis for band-limited binary phase-shift keying, when communicating over dispersive Nakagami- $m$ channels, although no direct sequence spreading was used.

In [10], the authors derived a new BER expression for coherent BPSK demodulation in a multipath Nakagami- $m$ fading asynchronous CDMA channel. The analysis assumed an arbitrary number of independent, but nonidentical resolvable paths combined by the RAKE receiver.

In [7], Yang and Hanzo investigated the performance of generalized multicarrier DS-CDMA [2] using various timelimited chip-waveforms, namely the rectangular, half-sine, as well as the raised-cosine chip-waveforms, and closed-form formulae were derived for evaluating the BER performance, when communicating over a dispersive Nakagami- $m$ channel.

Based on the above-mentioned studies, in this paper we investigate the performance of band-limited DS-CDMA systems in conjunction with different chip-waveform designs, when communicating over a dispersive Nakagami- $m$ channel. The required bandwidth $W$ of a DS-CDMA communication system is $W=\frac{1+\beta}{2 T_{c}}=\frac{G(1+\beta)}{2 T_{d}}$, where $T_{c}$ and $T_{d}$ represent the chip duration and data symbol duration respectively, while $\beta$ is the Nyquist roll-off factor, which determines the excess bandwidth and $G$ is the processing gain. It is plausible that a system using different chip-waveforms requires a different excess bandwidth. For example, the third generation wideband-CDMA system [11] employs square-root raised cosine spectral-domain shaping having a Nyquist roll-off factor of $\beta=0.22^{1}$. Furthermore, in the context of band-limited chip-waveforms, the interference factor associated with a specific pulse shape is expected to decrease as the excess bandwidth $\beta$ increases. In other words, we are capable of reducing the MAI imposed on other users at the cost of decreasing the attainable spectral efficiency, as it will be demonstrated in Section IV. It is possible to strike a trade-off between the processing gain $G$ and the excess bandwidth $\beta$. Hence in this paper we investigated the design of band-limited DS-CDMA systems, as a function of the processing gain $G$ and the excess bandwidth $\beta$. More specifically, owing to the fact that we have $G(1+\beta)=$ $2 W T_{d}$, we will investigate how to harmonize the processing

\footnotetext{
${ }^{1}$ Since the Nyquist roll-off factor $\beta$ and the excess bandwidth are directly linked to each other, both terminologies will be used interchangeable throughout this paper. This is justified, because we will be investigating not only Nyquist signalling, but a range of other bandlimited signalling pulses.
} 
gain $G$ and the excess bandwidth $\beta$ for the sake of achieving a good performance, when the system's bandwidth and the bit rate are fixed.

This paper is organized as follows. Section II describes the CDMA system designed for communicating over a dispersive Nakagami- $m$ channel, while Section III will analyze the BER performance of the band-limited DS-CDMA system in conjunction with different waveforms, which include both timelimited and band-limited chip-waveforms. Finally, Section IV provides our numerical results and Section V offers our conclusions.

\section{System Model}

\section{A. System Model}

Let us consider an asynchronous $K$-user DS-CDMA communication system, where each user is assigned a unique signature waveform $\mathbf{c}_{k}(t)=\sum_{i=0}^{G-1} c_{k i} \psi_{T_{c}}\left(t-i T_{c}\right)$. The sequence $c_{k i} \in \frac{1}{\sqrt{N}}\{+1,-1,+j,-j\}$ represents the random PseudoNoise (PN) spreading sequence of the $k$ th user and $G$ is the processing gain, which obeys $G=T_{d} / T_{c}$, and $\psi_{T_{c}}(t)$ represents the chip-waveform having an energy of $\int_{-\infty}^{+\infty} \psi_{T_{c}}^{2}(t) d t=$ $T_{c}$. For convenience, we define the normalized chip-waveform $\hat{\psi}(t)=\psi_{T_{c}}\left(t / T_{c}\right)$. For the band-limited system considered, the normalized chip-waveform $\hat{\psi}(t)$ satisfies:

$$
\int_{-\infty}^{+\infty} \hat{\psi}\left(t-n_{1}\right) \hat{\psi}^{*}\left(t-n_{2}\right) d t=\delta\left(n_{1}, n_{2}\right)
$$

where $\delta\left(n_{1}, n_{2}\right)=1$ for $n_{1}=n_{2}$, and 0 for $n_{1} \neq n_{2}$. From Eq.(1), we have:

$$
\sum_{n=-\infty}^{\infty} \hat{\psi}^{2}(n)=1
$$

Consequently, when the $K$ users' signals are transmitted over a frequency-selective fading channel, the received complexvalued low-pass equivalent signal at a given base station can be expressed as:

$$
\begin{aligned}
R(t)=\sum_{k=1}^{K} \sum_{l=0}^{L_{p}-1} \sqrt{2 P_{k}} \mathbf{c}_{k}\left(t-l T_{c}-\tau_{k}\right) \\
b_{k}\left(t-l T_{c}-\tau_{k}\right) h_{k l} \exp \left(j \theta_{k l}\right)+N(t),
\end{aligned}
$$

where $N(t)$ is the complex-valued low-pass-equivalent AWGN having a double-sided spectral density of $N_{0}$ and $\tau_{k}$ is the propagation delay of user $k$, while $L_{p}$ is the total number of resolvable paths.

\section{B. Channel Model}

The DS-CDMA signal experiences independent frequencyselective Nakagami- $m$ fading. The complex low-pass equivalent representation of the Channel Impulse Response (CIR) encountered by the $k$ th user is given by [8]:

$$
h_{k}(t)=\sum_{l=0}^{L_{p}-1} h_{k l} \delta\left(t-l T_{c}\right) \exp \left(j \theta_{k l}\right),
$$

where $h_{k l}$ represents the Nakagami-distributed fading envelope, $l T_{c}$ is the relative delay of the $l$ th path of user $k$ with respect to the main path, while $L_{p}$ is the total number of resolvable multipath components. Furthermore, $\theta_{k l}$ is the uniformly distributed phase-shift of the $l$ th multipath component of the channel and $\delta(t)$ is the Kronecker Delta-function. More explicitly, the $L$ multipath attenuations $\left\{h_{k l}\right\}$ are independent Nakagami distributed random variables having a Probability Density Function (PDF) of [12]-[14]:

$$
\begin{aligned}
p\left(h_{k l}\right) & =M\left(h_{k l}, m_{k l}, \Omega_{k l}\right), \\
M(R, m, \Omega) & =\frac{2 m^{m} R^{2 m-1}}{\Gamma(m) \Omega^{m}} e^{(-m / \Omega) R^{2}},
\end{aligned}
$$

where $\Gamma(\cdot)$ is the gamma function [8], and $m_{k l}$ is the Nakagami- $m$ fading parameter, which characterizes the severity of the fading for the $l$-th resolvable path of user $k$ [15] . Specifically, $m_{k l}=1$ represents Rayleigh fading, $m_{k l} \rightarrow$ $\infty$ corresponds to the conventional Gaussian scenario and $m_{k l}=1 / 2$ describes the so-called one-sided Gaussian fading, i.e. the worst-case fading condition. The Ricean and lognormal distributions can also be closely approximated by the Nakagami distribution in conjunction with values of $m_{k l}>$ 1. The parameter $\Omega_{k l}$ in Eq.(5) is the second moment of $h_{k l}$, i.e. we have $\Omega_{k l}=E\left[\left(h_{k l}\right)^{2}\right]$. We assume a negative exponentially decaying multipath intensity profile (MIP) given by $\Omega_{k l}=\Omega_{k 0} e^{-\eta l}, \eta \geq 0, l=0, \ldots, L_{p}-1$, where $\Omega_{k l}$ is the average signal strength corresponding to the first resolvable path, where $\Omega_{k 0}$ is the average single strength of the first resolvable path, and $\eta$ is the rate of average power decay.

\section{BER ANALYSIS}

Let the first user be the user-of-interest and consider a receiver using de-spreading as well as multipath diversity combining. The conventional matched filter based RAKE receiver using MRC may be invoked for detection, where we assume that the RAKE receiver is capable of combining $L_{r}$ number of diversity paths.

Let us assume that we have achieved time synchronization and perfect estimates of the channel magnitudes and phases are available. The individual matched filter outputs are appropriately delayed, in order to coherently combine the $L_{r}$ number of path signals processed by the RAKE combiner. The $l$ th RAKE combiner finger's output $Z_{k l}$ is sampled at $t=T+l T_{c}+\tau_{k}$, in order to detect the $k$ th user's transmitted symbol $b_{k}[0]$, which is expressed as:

$$
Z_{k l}=D_{k l}+I_{k l}
$$

where $D_{k l}$ represents the desired direct Line-of-Sight (LOS) component, which can be expressed as:

$$
D_{k l}=\sqrt{2 P} T_{s} b_{k}[0] h_{k l}^{2} .
$$

In Eq.(7) $b_{k}[0]$ is the first bit transmitted by the $k$ th BPSK user and we have $b_{k}[0] \in\{+1,-1\}$. Hence, the interference plus noise term $I_{k}$ in Eq.(6) may be expressed as:

$$
I_{k l}=I_{k l}[S]+I_{k l}[M]+N_{k l}
$$


where $I_{k l}[S]$ represents the multipath interference imposed by the user-of-interest. Explicitly, $I_{k l}[S]$ may be expressed as:

$$
\begin{aligned}
I_{k l}[S]=\sqrt{2 P} T_{s} h_{k l} \sum_{\substack{l_{p}=0 \\
l_{p} \neq l}}^{L_{p}-1} h_{k l_{p}} \exp \left(j \theta_{k l_{p}}\right) & \left\{\sum_{n=-\infty}^{\infty} \rho_{k k}(n) \hat{\psi}(n)\right\},
\end{aligned}
$$

where the term $\rho_{k k}(n)$ is formulated as:

$$
\rho_{k k}(n)=\frac{1}{G} \sum_{i=0}^{G-1} c_{k}[i]^{*} c_{k}\left[i-n G-l_{p}\right] .
$$

Furthermore, $I_{k l}[M]$ of Eq.(8) represents the multiuser interference inflicted by the $K-1$ interfering users, which is expressed as:

$$
\begin{aligned}
I_{k l}[M]= & \sqrt{2 P} T_{s} h_{k l} \sum_{\substack{k^{\prime}=1 \\
k^{\prime} \neq k}}^{K} \sum_{l_{p}=0}^{L_{p}-1} h_{k^{\prime} l_{p}} \exp \left(j \theta_{k l_{p}}\right) \\
& \times\left\{\sum_{n=-\infty}^{\infty} \rho_{k k^{\prime}}(n) \hat{\psi}\left(n-\tau_{k}\right)\right\},
\end{aligned}
$$

and we have:

$$
\rho_{k k^{\prime}}(n)=\frac{1}{G} \sum_{i=0}^{G-1} c_{k}[i]^{*} c_{k^{\prime}}\left[i-n G-l_{p}\right]
$$

It was shown in [5], [16] for a random PN spreading sequence that the random variables $\rho_{k k}(n)$ and $\rho_{k k^{\prime}}(n)$ may be modelled as complex Gaussian random variables having a mean of zero and a variance of $1 / G$. Therefore, the variance of the term $I_{k l}[S]$ of Eq.(8), which was explicitly formulated in Eq.(9) can be expressed as:

$\operatorname{Var}\left\{I_{k l}[S]\right\}=2 P T_{s}^{2} h_{k l}^{2} \Omega_{0}\left[q\left(L_{p}, \eta\right)-1\right] \frac{1}{G} \sum_{n=-\infty}^{\infty} \hat{\psi}^{2}(n)$,

where $q\left(L_{p}, \eta\right)=\sum_{l=0}^{L_{p}-1} e^{-\eta l}$, the above equation may be simplified with the aid of Eq.(2) to:

$$
\operatorname{Var}\left\{I_{k l}[S]\right\}=2 P T_{s}^{2} h_{k l}^{2} \frac{1}{G} \Omega_{0}\left[q\left(L_{p}, \eta\right)-1\right] .
$$

Similarly, the variance of the term $I_{k l}[M]$ formulated in Eq.(11) can be expressed as Eq. (15), which is found at the top of the next page, where $\hat{\psi}^{2}(n-\tau)=\int_{0}^{T_{c}} \hat{\psi}(t) \hat{\psi}^{*}(t+n-\tau) d t$ and $E[\phi(\beta, \tau)]=E\left[\sum_{n=-\infty}^{\infty} \hat{\psi}^{2}(n-\tau)\right]$ defines the interference factor associated with a specific chip pulse shape, which predetermines the amount of the MAI imposed by different chip-waveforms, while $\tau$ is a random variable uniformly distributed in $[0,1]$, and $E[\phi(\beta, \tau)]$ can be expressed as a function of the excess bandwidth $\beta$, as we mentioned before.

Finally, the noise term of Eq.(8) can be expressed as:

$$
N_{k l}=h_{k l} \int_{0}^{T_{s}} n(t) c[t] d t
$$

which is a Gaussian random variable having zero mean and a variance of $2 N_{0} T_{s} h_{k l}^{2}$.

The MRC's decision variable $Z_{k}$, is constituted by the sum of the RAKE fingers' output, which can be expressed as:

$$
Z_{k}=\sum_{l=0}^{L_{r}-1} Z_{k l}
$$

\section{A. Probability of Error}

In the analysis of this section we employ the Gaussian approximation and hence model both the multiuser interference and the self-interference terms of Eq.(8) as an AWGN process having zero mean and a variance equal to the corresponding variances. More explicitly, the assumptions made in this section are as follows. The RAKE fingers' output signal $Z_{k l}$ is a Gaussian distributed random variable having a mean of $D_{k l}$. Consequently, according to the analysis of the previous sections provided for the random spreading codes and BPSK modulation considered, the variance of the $l$ th RAKE finger's output samples $Z_{k l}$ for a given set of channel amplitudes $\left\{h_{k l}\right\}$ may be approximated as Eq. (18), found on the next page, where $E_{b}=P T_{s}$ is the energy per bit. Furthermore, the MRC's output sample $Z_{k}$ can be approximated by an AWGN variable having a mean value of $\mathrm{E}\left[Z_{k}\right]=\sum_{l=0}^{L_{r}-1} D_{k l}$ and a variance of $\operatorname{Var}\left[Z_{k}\right]=\sum_{l=0}^{L_{r}-1} \sigma_{k l}$ [13], [17]. To expound further, upon using Eq.(7) and Eq.(18) we have:

$$
\mathrm{E}\left[Z_{k}\right]=\sum_{l=0}^{L_{r}-1} \sqrt{2 P} T_{s} b_{k}[0] h_{k l}^{2}
$$

and Eq. (20), which is found on the top of the next page. Therefore, the BER of BPSK modulation conditioned on the set of fading magnitudes $\left\{h_{k l}, l=0,1, \ldots, L_{r}-1\right\}$ can be expressed as:

$$
P_{b}(\gamma)=Q\left(\sqrt{\frac{\left(\mathrm{E}\left[Z_{k}\right]\right)^{2}}{\operatorname{Var}\left[Z_{k}\right]}}\right)=Q\left(\sqrt{\sum_{l=0}^{L_{r}-1} 2 \gamma_{l}}\right)
$$

where $Q(x)$ represents the Gaussian $Q$-function, which can also be represented in its less conventional form as [15], [17] $Q(x)=\frac{1}{\pi} \int_{0}^{\pi / 2} \exp \left(-\frac{x^{2}}{2 \sin ^{2} \theta}\right) d \theta$, where $x \geq 0$. Furthermore, $2 \gamma_{l}$ in Eq.(21) represents the output Signal to Interference plus Noise Ratio (SINR) at the $l$ th finger of the RAKE receiver, while $\gamma_{l}$ is given by:

$$
\gamma_{l}=\gamma_{c} \cdot \frac{h_{k l}^{2}}{\Omega_{0}} .
$$

Let us now substitute Eq.(22) into Eq.(21) and Eq.(19) as well as Eq.(20) into Eq.(22). We can see then that the expressions under the square-root functions must be equal, which allows us to express $\gamma_{c}$ as follows:

$$
\begin{aligned}
& \gamma_{c}= \\
& {\left[\frac{K q\left(L_{p}, \eta\right) E[\phi(\beta, \tau)]}{G}+\frac{q\left(L_{p}, \eta\right)-1}{G}+\left(\frac{\Omega E_{b}}{N_{0}}\right)^{-1}\right]^{-1}}
\end{aligned}
$$




$$
\operatorname{Var}\left\{I_{k l}[M]\right\}=2 P T_{s}^{2} h_{k l}^{2} \Omega_{0} K q\left(L_{p}, \eta\right) \frac{1}{G} E\left[\sum_{n=-\infty}^{\infty} \hat{\psi}^{2}(n-\tau)\right]=2 P T_{s}^{2} h_{k l}^{2} \Omega_{0} K q\left(L_{p}, \eta\right) \frac{1}{G} E[\phi(\beta, \tau)]
$$

$$
\sigma_{k l}^{2}=\frac{1}{2} \times\left\{\operatorname{Var}\left\{I_{k l}[S]\right\}+\operatorname{Var}\left\{N_{k l}\right\}+\operatorname{Var}\left\{I_{k l}[N]\right\}\right\}=2 P T_{s}^{2}\left[\frac{K q\left(L_{p}, \eta\right) E[\phi(\beta, \tau)]}{2 G}+\frac{q\left(L_{p}, \eta\right)-1}{2 G}+\left(\frac{2 \Omega_{0} E_{b}}{N_{0}}\right)^{-1}\right] \cdot \Omega_{0} h_{k l}^{2}
$$

$$
\operatorname{Var}\left[Z_{k}\right]=2 P T_{s}^{2}\left[\frac{K q\left(L_{p}, \eta\right) E[\phi(\beta, \tau)]}{2 G}+\frac{q\left(L_{p}, \eta\right)-1}{2 G}+\left(\frac{2 \Omega_{0} E_{b}}{N_{0}}\right)^{-1}\right] \cdot \Omega_{0} \sum_{l=0}^{L_{r}-1} h_{k l}^{2}
$$

The average BER $P_{b}(E)$ at a given value of $E_{b} / N_{0}$ can be obtained by the weighted averaging of the output $\gamma_{l}$, i.e. upon integrating the conditional BER of Eq.(21) after weighting it by the probability of occurrence of a specific instantaneous value of $\gamma_{l}$, which is quantified by the joint PDF of the instantaneous $\gamma_{l}$ values corresponding to the $L_{r}$ multipath components $\left\{\gamma_{l}: l=0,2, \ldots, L_{r}-1\right\}$. Since the random variables $\left\{\gamma_{l}: l=1,2, \ldots, L_{r}-1\right\}$ are assumed to be statistically independent, the average BER expressed in Eq.(21) can be formulated as [18]:

$$
P_{b}(E)=\frac{1}{\pi} \int_{0}^{\pi / 2} \prod_{l=0}^{L_{r}-1} I_{l}\left(\bar{\gamma}_{l}, \theta\right) d \theta
$$

where $I_{l}\left(\bar{\gamma}_{l}, \theta\right)$ is given by:

$$
I_{l}\left(\bar{\gamma}_{l}, \theta\right)=\int_{0}^{\infty} \exp \left(-\frac{\gamma_{l}}{\sin ^{2} \theta}\right) p_{\gamma_{l}}\left(\gamma_{l}\right) d \gamma_{l} .
$$

Since we have $\gamma_{l}=\gamma_{c} \cdot \frac{h_{l}^{2}}{\Omega_{0}}$ and $h_{l}$ obeys the Nakagami- $m$ distribution characterized by Eq.(5), it can be shown that the PDF of $\gamma_{l}$ can be formulated as [15], [18]:

$$
p_{\gamma_{l}}\left(\gamma_{l}\right)=\left(\frac{m}{\bar{\gamma}_{l}}\right)^{m} \frac{\gamma^{m-1}}{\Gamma(m)} \exp \left(-\frac{m \gamma_{l}}{\bar{\gamma}_{l}}\right), \quad \gamma_{l} \geq 0,
$$

where $\bar{\gamma}_{l}=\gamma_{c} e^{-\eta l}$ for $l=0,1, \ldots, L_{r}-1$.

Upon substituting Eq.(26) into Eq.(25) it can be shown that we have [15]:

$$
I_{l}\left(\bar{\gamma}_{l}, \theta\right)=\left(\frac{m \sin ^{2} \theta}{\bar{\gamma}_{l}+m \sin ^{2} \theta}\right)^{m} .
$$

Finally, upon substituting Eq.(27) into Eq.(24), the average BER of the CDMA system considered can be written as:

$$
P_{b}(E)=\frac{1}{\pi} \int_{0}^{\pi / 2} \prod_{l=0}^{L_{r}-1}\left(\frac{m \sin ^{2} \theta}{\bar{\gamma}_{l}+m \sin ^{2} \theta}\right)^{m} d \theta .
$$

\section{B. Time-Limited Waveforms}

In this section, we will consider three widely-used timelimited chip-waveforms, which are the rectangular, half-sine and raised-cosine time-domain chip-waveforms, respectively. Explicitly, the time domain shape of these waveforms is formulated as follows.

1) Rectangular:

$$
\psi_{T_{c}}^{r}(t)=u(t)
$$

2) Half-Sine:

$$
\psi_{T_{c}}^{s}(t)=\sqrt{2} \sin \left(\frac{\pi t}{T_{c}}\right) u(t)
$$

3) Raised Cosine:

$$
\psi_{T_{c}}^{c}(t)=\sqrt{\frac{2}{3}}\left[1-\cos \left(\frac{2 \pi t}{T_{c}}\right)\right] u(t) . \text { The corresponding }
$$

frequency-domain spectral density function $Q(f)$ may be expressed with the aid of the Fourier transform $Q(f)=$ $\mathcal{F}\left\{\psi_{T_{c}}(t)\right\}$. More specifically, the spectral density function $Q(f)$ of these waveforms can be expressed as a function of the normalized frequency $f T_{c}$, and for notational convenience we denote the normalized frequency $f T_{c}$ as $f$. Then we have:

1) Rectangular:

$$
Q^{r}(f)=\operatorname{sinc}(f)
$$

2) Half-Sine:

$$
Q^{s}(f)=\frac{\cos (\pi f)}{1-4(f)^{2}}
$$

3) Raised Cosine:

$$
Q^{c}(f)=\operatorname{sinc}(f)+\frac{1}{2} \operatorname{sinc}(f-1)+\frac{1}{2} \operatorname{sinc}(f+1) .
$$

We considered two different fractional energy containment bandwidth definitions [1], [6] for the sake of characterizing these time-limited waveforms. It is conceptually appealing to define the fractional energy containment bandwidth [1] as the normalized frequency $W$ that satisfies $\frac{\int_{-W}^{W} Q^{2}(f) d f}{\int_{-\infty}^{\infty} Q^{2}(f) d f} \geq$ $99 \%$. In [1], the similar but more stringent definition of $\frac{\int_{-W}^{W} Q^{2}(f) d f}{\int_{-\infty}^{\infty} Q^{2}(f) d f} \geq 99.995 \%$ was used, especially in the context of finite time duration signalling pulses. In terms of the Nyquist excess bandwidth $\beta$, we have:

$$
\beta=2 W T_{c}-1 \text {. }
$$

In [4], [5], Dallas and Pavlidou investigated the interference factor, $E[\phi(\beta, \tau)]$ defined in the context of Eq.(15), which was found to be $0.666,0.596$ and 0.582 for the rectangular, halfsine and raised cosine chip-waveforms, respectively. Therefore, following Eq.(29) and the above definition of the energy containment bandwidth, the required excess bandwidths are summarized in Table I for both of the above mentioned energy containment factors, along with the corresponding interference factor for the three time-limited chip-waveforms considered.

\section{Band-Limited Waveforms}

Having studied three time-limited waveforms in the previous section, in this section we will consider two different bandlimited waveforms. The first is the well-known frequencydomain raised cosine Nyquist spectrum [8], where $\beta$ represents the Nyquist roll-off factor, quantifying the excess 
TABLE I

THE REQUIRED EXCESS BANDWIDTH $\beta$ AND THE CORRESPONDING interference factor $E[\phi(\beta, \tau)]$ FOR VARIOUS TIME-DOMAIN CHIP-PULSE SHAPES

\begin{tabular}{|l|l|l|l|}
\hline Waveform & $\begin{array}{l}\text { Energy containment } \\
\text { bandwidth } \geq 99 \%\end{array}$ & $\begin{array}{l}\text { Energy containment } \\
\text { bandwidth } \geq 99.995 \%\end{array}$ & $\begin{array}{l}\text { Interference } \\
\text { factor } E[\phi(\beta, \tau)]\end{array}$ \\
\hline Rectangular & $\beta=12.22$ & $\beta=38.34$ & 0.666 \\
\hline Half-Sine & $\beta=1.36$ & $\beta=12.90$ & 0.596 \\
\hline Raised Cosine & $\beta=1.82$ & $\beta=5.58$ & 0.482 \\
\hline
\end{tabular}

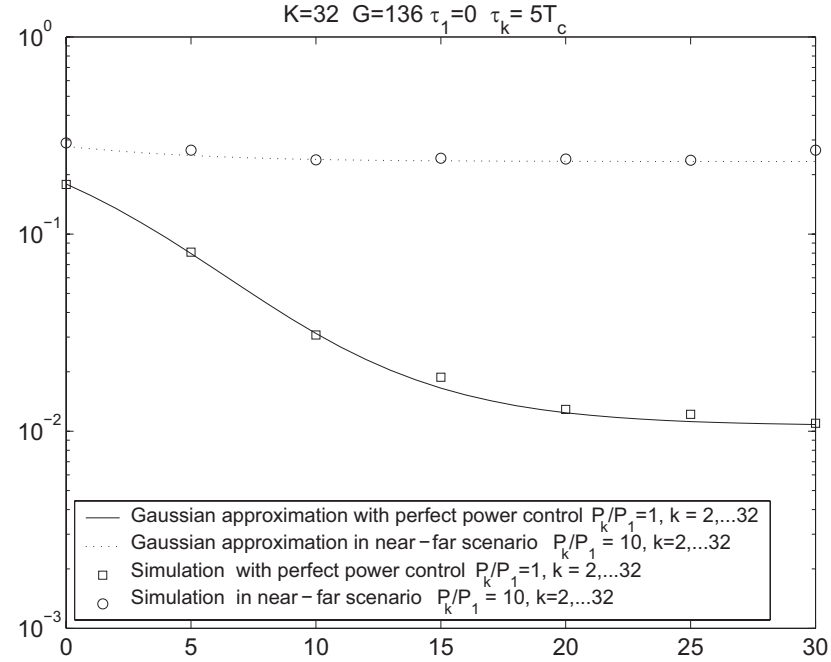

Fig. 1. Comparison of the simulated and theoretical performance using the Gaussian approximation for transmission over an asynchronous AWGN channel in both a perfectly power controlled and in a severe near-far scenario, where the system parameters were $G=136, K=32, \tau_{1}=0$ and $\tau_{k}=5 T_{c}$.

Nyquist bandwidth. We will refer to this Nyquist signalling pulse as a band-limited Raised Cosine (BRC) signalling waveform, which is characterized by the spectral domain representation given by [19]:

$|Q(f)|^{2}= \begin{cases}0, & \text { for }|f| \geq \frac{1+\beta}{2} \\ 1, & \text { for }|f| \leq \frac{1-\beta}{2} \\ \frac{1}{2}\left\{1+\cos \left(\frac{\pi}{\beta}\left(|f|-\frac{1}{2}\right)\right)\right\}, & \text { elsewhere. }\end{cases}$

Another band-limited chip-waveform is the so-called optimum waveform $[5]^{2}$, which was proposed by Cho and Lehnert [5] for the BER performance analysis of band-limited DSCDMA systems communicating over an AWGN channels, which is given by:

$$
|Q(f)|^{2}= \begin{cases}1, & \text { for }|f|<\frac{1-\beta}{2} \\ \frac{1}{2}, & \text { for } \frac{1-\beta}{2} \leq|f| \leq \frac{1+\beta}{2} \\ 0, & \text { elsewhere. }\end{cases}
$$

Cho and Lehnert [5] investigated the associated interference factor $E[\phi(\beta, \tau)]$ for both the BRC waveform and for the optimum waveform. Explicitly, they found that the interference factor of the pulse shape for the BRC waveform can be

\footnotetext{
${ }^{2}$ The terminology 'optimum' implies in this context that the best BER performance was achieved by the system considered, when communicating over an AWGN channel.
}

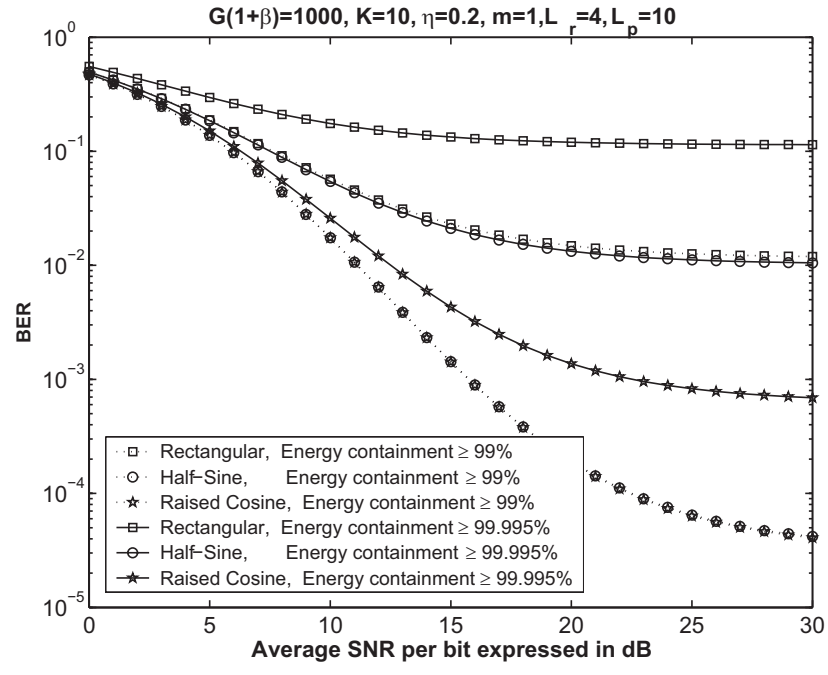

Fig. 2. BER performance comparisons of various band-limited DS-CDMA systems as a function of $E_{b} / N_{0}$ when communicating over a Rayleigh channel associated with $m=1$ in conjunction with three time-limited chip-waveforms, where two different energy containment factors, namely $\geq 99 \%$ and $\geq 99.995 \%$ were considered. A negative-exponentially decaying Multipath Intensity Profile (MIP) having a decay factor of $\eta=0.2$ was used, the total number of resolvable paths was $L_{p}=10$, but the RAKE-receiver's complexity was limited to combining only $L_{r}=4$ paths. A total of $K=10$ users were supported.

expressed as [5]:

$$
\begin{aligned}
\phi(\beta, \tau) & =1-\frac{\beta}{4}+\frac{\beta}{4} \cos (2 \pi \tau), \\
E[\phi(\beta, \tau)] & =1-\frac{\beta}{4},
\end{aligned}
$$

while that for the optimum bandlimited waveform as [5]:

$$
\begin{aligned}
\phi(\beta, \tau) & =1-\frac{\beta}{2}+\frac{\beta}{2} \cos (2 \pi \tau), \\
E[\phi(\beta, \tau)] & =1-\frac{\beta}{2} .
\end{aligned}
$$

Having considered the associated interference factor of the BRC and optimum chip-pulse shape for the family of the band-limited waveforms, in next section we will consider the optimization of the processing gain $G$ and the excess bandwidth $\beta$.

\section{Jointly Optimizing the Processing Gain $G$ and the Excess Bandwidth $\beta$ for band-limited Waveforms}

Given a bandwidth $W$ and a transmitted bit rate of $\frac{1}{T_{d}}$, according to Eq.(23) we have to minimize $\frac{E[\phi(\beta, \tau)]}{G}$ under the constraint that the total system's bandwidth of $G(1+\beta)=$ $2 W T_{d}$ is a constant. More explicitly, the inteference factor 


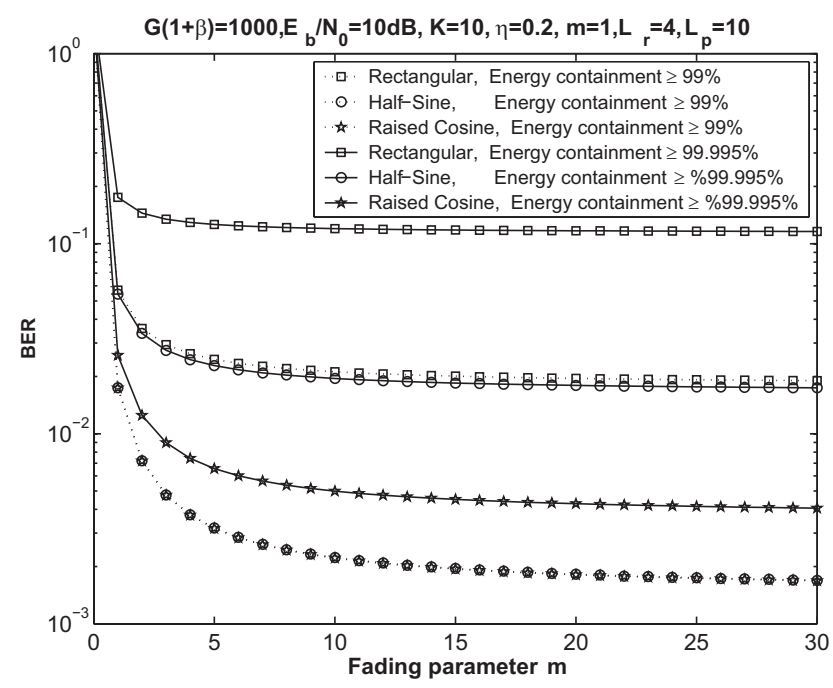

Fig. 3. BER performance comparisons of various band-limited DS-CDMA systems as a function of the Nakagami fading parameter $m$ in conjunction with three time-limited chip-waveforms, where two different energy containment factors, namely $\geq 99 \%$ and $\geq 99.995 \%$ were considered. A negative-exponentially decaying Multipath Intensity Profile (MIP) having a decay factor of $\eta=0.2$ was used, the total number of resolvable paths was $L_{p}=10$, but the RAKE-receiver's complexity was limited to combining only $L_{r}=4$ paths. A total of $K=10$ users were supported.

$E[\phi(\beta, \tau)]$ is divided by $G$ for the sake of optimising the system, because the increased amount of MAI imposed by a chip-waveform having higher spectral-domain side-lobes manifests itself in terms of an increased interference factor. The effects of this increased MAI maybe compensated by employing a higher spreading factor or spreading gain, hence their joint optimum is sought. This optimisation problem can be solved with the aid of Lagrange multipliers. Hence for the sake of finding the optimum bandlimited chip-waveform, let

$$
\Omega(G, \beta)=\frac{E[\phi(\beta, \tau)]}{G}+\lambda\left[G(1+\beta)-2 W T_{d}\right]
$$

Then, upon using the optimum bandlimited waveform of Cho and Lehnert [5], we have:

$$
\Omega(G, \beta)=\frac{1-\frac{\beta}{2}}{G}+\lambda\left[G(1+\beta)-2 W T_{d}\right] .
$$

Owing to the fact that $\Omega(G, \beta)$ is a strictly convex function of $G$ and $\beta$, for the optimum waveform the worst possible configuration of the variables $G$ and $\beta$ satisfies:

$$
\begin{aligned}
& \frac{\partial \Omega(G, \beta)}{\partial G}=-\frac{1-\beta / 2}{G^{2}}+\lambda(1+\beta)=0, \\
& \frac{\partial \Omega(G, \beta)}{\partial \beta}=-\frac{1}{2 G}+\lambda G=0,
\end{aligned}
$$

and from Eq.(36) we arrive at the maximum of $\Omega(G, \beta)$ for $\beta=1 / 2$.

Similarly, for the BRC waveform it may be readily shown that a choice of $\beta=3 / 2$ achieved the worst-case performance. However, in our case $\beta$ is limited to the interval of $[0,1]$, hence $\beta=1$ achieved the worst possible performance in the context of the BRC waveform. In the next section we will discuss the choice of $\beta$ required for achieving the best possible performance for band-limited chip-waveform based DS-CDMA systems.

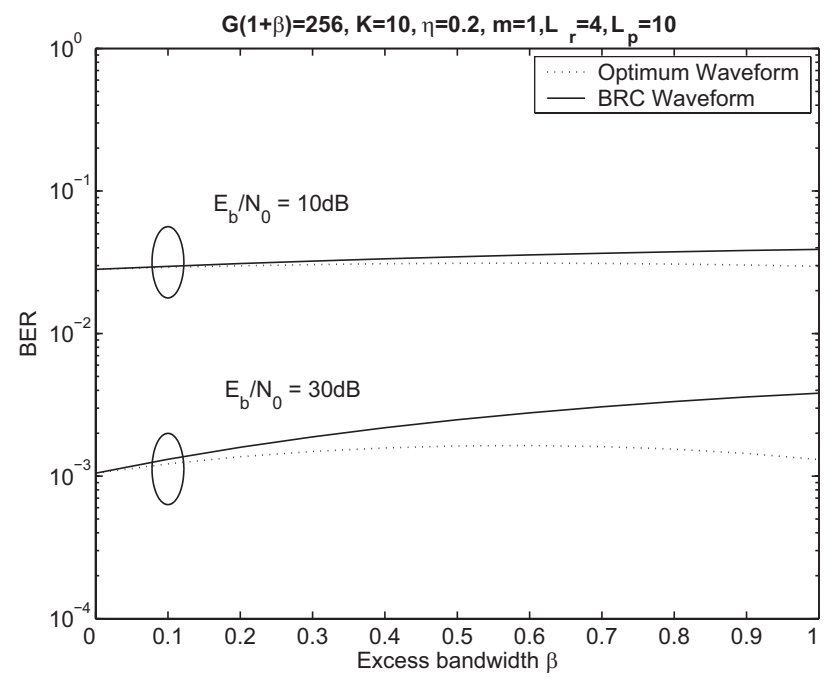

Fig. 4. BER performance comparison of band-limited DS-CDMA as a function of the excess bandwidth $\beta$ in conjunction with the BRC and the optimum waveforms [5], when the bandwidth required was fixed to $G(1+\beta)=256$. A negative-exponentially decaying Multipath Intensity Profile (MIP) having a decay factor of $\eta=0.2$ was used, the total number of resolvable paths was $L_{p}=10$, but the RAKE-receiver's complexity was limited to combining only $L_{r}=4$ paths, and a total of $K=10$ users were supported.

\section{NumericAl Results}

In this section we will investigate the achievable BER performance of band-limited DS-CDMA. As mentioned before, the normalized bandwidth can be expressed as $G(1+\beta)=$ $2 W T_{d}$. Hence upon stipulating a specific $G(1+\beta)$ value, the system's bandwidth as well as the bit rate has been fixed. The parameters employed in our investigations were $L_{p}=10, L_{r}=4, \eta=0.2$, and $K=10$.

We commence our performance-related discussions by validating the Gaussian approximation using simulations in two specific scenarios, namely in case of perfect power control, when all the users have an identical received signal power, as well as in a severe near-far-problem scenario, where the interfering users have a ten-times higher power than the reference user. As seen in Fig. 1 for transmission over an asynchronous AWGN channel, indeed, the Gaussian approximation is accurate in both the perfect power control scenario, as well as in the near-far scenario. Again, the accuracy of the Gaussian approximation was also corroborated by the authors of [9], who provided an exact error-rate analysis for bandlimited binary phase-shift keying, when communicating over dispersive Nakagami- $m$ channels, although no direct sequence spreading was used. It transpires that the Gaussian approximation constitutes an extremely accurate assumption, when the number of sources imposing interference becomes higer than eight, regardless of the specific individual distributions of the constituent random processes.

Fig. 2 portrays the attainable BER performance of DSCDMA in conjunction with the previously mentioned three time-limited waveforms at $G(1+\beta)=1000$. These results were plotted by evaluating Eq.(28). We can observe that in Fig. 2 the raised cosine waveform based DS-CDMA system is capable of achieving the best performance, since its frequency-domain spectral side-lobe is relatively low, and 


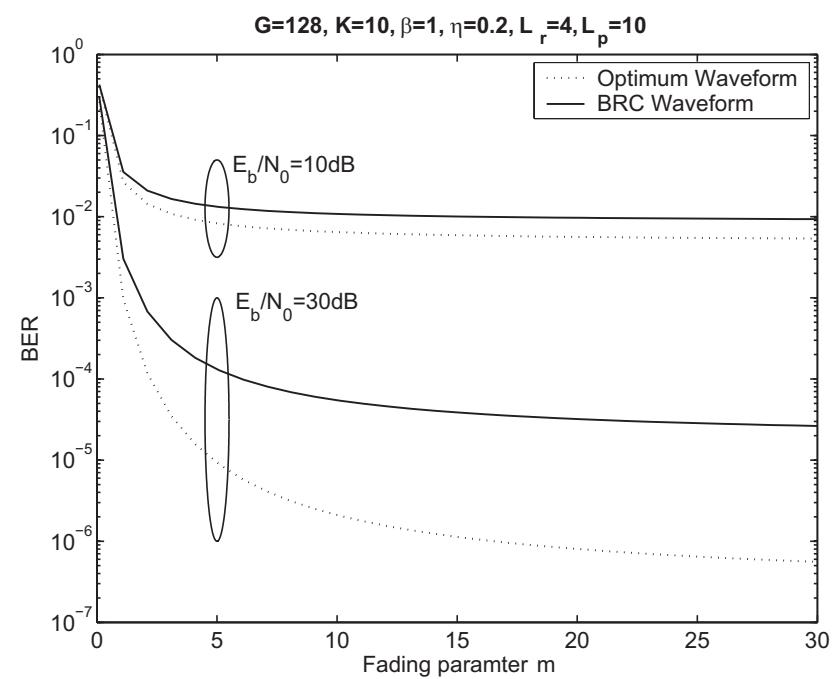

Fig. 5. BER performance comparison of band-limited DS-CDMA as a function of the Nakagami fading parameter $m$ in conjunction with the BRC and the optimum [5] waveforms, where the excess bandwidth was fixed to $\beta=1$, and the processing gain was $G=128$. A negative-exponentially decaying Multipath Intensity Profile (MIP) having a decay factor of $\eta=0.2$ was used, the total number of resolvable paths was $L_{p}=10$, but the RAKEreceiver's complexity was limited to combining only $L_{r}=4$ paths, and a total of $K=10$ users were supported. As $m$ increases, the channel becomes a dispersive AWGN-like transmission medium, resulting in a reduced BER.

the corresponding excess bandwidth values are listed in Table I. By contrast, the performance of the rectangular chipwaveform based DS-CDMA scheme is the worst amongst the three chip-waveforms investigated. When considering an energy containment in excess of $99 \%$, the half-sine signalling chip-waveform based DS-CDMA system achieves a similar performance to that of the scheme using a time-domain raised cosine chip-waveform. This is because as seen in Table I, the half-sine chip-waveform based DS-CDMA system requires a smaller excess bandwidth $\beta$ than that of the raised cosine chip-waveform based system, when the energy containment is in excess of $99 \%$, while at the same time exhibiting a higher interference factor of $E[\phi(\beta, \tau)]$. Fig. 3 portrays the attainable BER performance of band-limited DS-CDMA as a function of the Nakagami fading parameter $m$. From this figure we may infer similar conclusions to those accruing from Figure 2, when communicating over different fading channels characterised by various Nakagami fading parameters.

In Fig. 4 we investigated the trade-offs between the different values of the spreading gain $G$ and the excess bandwidth $\beta$ at a fixed bandwidth requirement of $G(1+\beta)=256$. From this figure we can observe that the BER performance of the optimum chip-waveform based DS-CDMA system is at its worst, when we have $\beta=0.5$, although this trend is only visible at $E_{b} / N_{0}=30 \mathrm{~dB}$. This performance trend is in line with our discussions provided in Section III-D. By contrast, when we have $\beta \rightarrow 0$ and $\beta \rightarrow 1$, the system is capable of achieving its best possible performance, although again, these trends are only perceivable at $E_{b} / N_{0}=30 \mathrm{~dB}$. Furthermore, for the BRC waveform, when we have $\beta \rightarrow 0$ the system attains its best possible performance. Fig. 5 comparatively studied the performance of the BRC waveform and the optimum chip-waveform of [5] as a function of the Nakagami

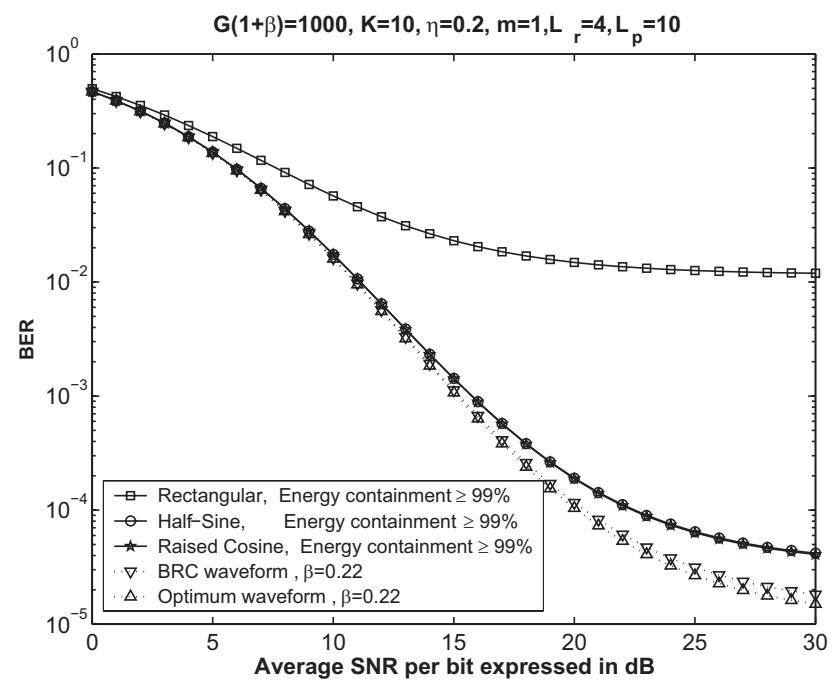

Fig. 6. BER performance comparison of band-limited DS-CDMA as a function of $E_{b} / N_{0}$ in conjunction with the three time-limited and two bandlimited chip-waveforms studied, when the required bandwidth was fixed to $G(1+\beta)=1000$. A negative-exponentially decaying Multipath Intensity Profile (MIP) having a decay factor of $\eta=0.2$ was used, the total number of resolvable paths was $L_{p}=10$, but the RAKE-receiver's complexity was limited to combining only $L_{r}=4$ paths, and a total of $K=10$ users were supported.

fading parameter $m$. From this figure we may conclude that the optimum waveform is capable of achieving a significantly better performance than that of the BRC waveform, when we have $\beta \rightarrow 1$.

In Fig. 6 we investigated the BER performance of bandlimited DS-CDMA in conjunction with the tree different time-limited and two band-limited chip-waveforms considered, when the bandwidth occupied was fixed to $G(1+\beta)=1000$. From this figure we infer that a frequency-domain BRC waveform based DS-CDMA system employing $\beta=0.22$ is capable of achieving a similar performance to that of using the optimum waveform [5] associated with $\beta=0.22$, when communicating over a Rayleigh fading channel. Furthermore, the time-domain raised-cosine waveform aided CDMA system of Fig. 6 was also capable of approaching the BER of both the frequency-domain BRC and optimum chip-waveform, although at $E_{b} / N_{0}=30 \mathrm{~dB}$ it exhibited an approximately factor two higher residual BER. Finally, Fig. 7 portrays the BER performance of the band-limited DS-CDMA system investigated as a function of the number of users supported.

\section{CONCLUSiON}

In this paper, we have investigated the achievable performance of band-limited DS-CDMA in conjunction with three different time-limited and two band-limited chip-waveforms. In the context of the time-limited waveforms the raised cosine waveform based DS-CDMA system achieved the best performance, because its frequency-domain spectral side-lobe is relatively low. By contrast, when we considered bandlimited waveforms, we investigated the BER performance of both the optimum [5] and BRC waveform based DS-CDMA systems. Both of these band-limited waveform based DSCDMA schemes exhibited a better BER performance than that of the time-limited waveforms. When aiming for an energy 


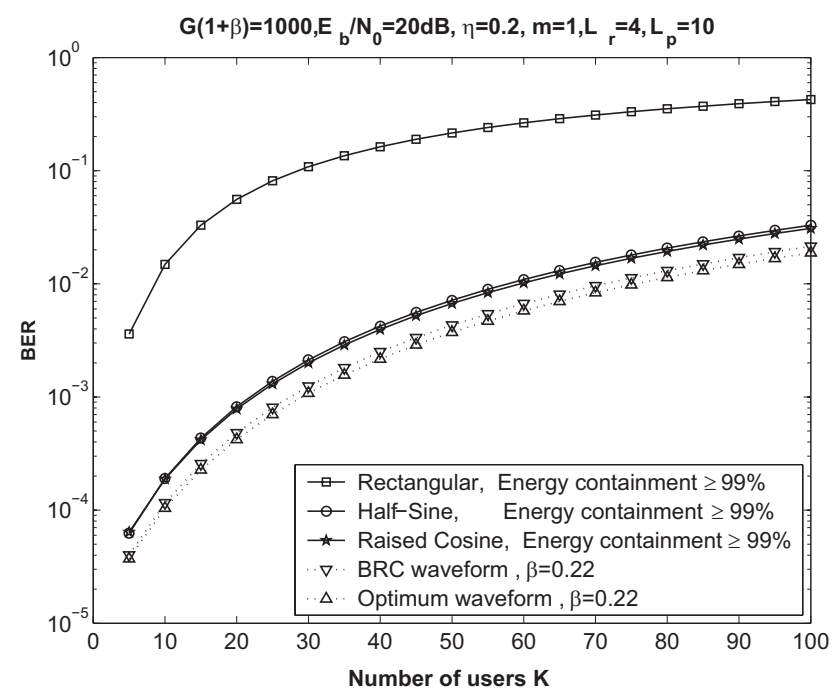

Fig. 7. BER performance comparison of band-limited DS-CDMA as a function of the number of users $K$ supported in conjunction with the three time-limited and two band-limited chip-waveforms studied, when the required bandwidth was fixed to $G(1+\beta)=1000$. A negative-exponentially decaying Multipath Intensity Profile (MIP) having a decay factor of $\eta=0.2$ was used, the total number of resolvable paths was $L_{p}=10$, but the RAKE-receiver's complexity was limited to combining only $L_{r}=4$ paths, and the SNR of each user was fixed to $E_{b} / N_{0}=20 \mathrm{~dB}$.

containment in excess of $99 \%$, the raised cosine waveform based DS-CDMA scheme was capable of achieving a similar performance to that of the optimum waveform based DSCDMA arrangement.

\section{REFERENCES}

[1] F. Amoroso, "The bandwidth of digital data signals," IEEE Commun. Mag., vol. 18, pp. 13-24, Nov. 1980.

[2] L. Hanzo, L. L. Yang, E. L. Kuan, and K. Yen, Single- and Multi-Carrier DS-CDMA. New York: John Wiley and IEEE Press, 2003.

[3] M. A. Landolsi and W. E. Stark, "DS-CDMA chip waveform design for minimal interference under bandwidth, phase, and envelope constraints," IEEE Trans. Commun., vol. 47, pp. 1737-1746, Nov. 1999.
[4] P. I. Dallas and F.-N. Pavlidou, "Innovative chip waveforms in microcelluar DS/CDMA packet mobile radio," IEEE Trans. Commun., vol. 44, pp. 1413-1416, Nov. 1996.

[5] J. H. Cho and J. S. Lehnert, "An optimal signal design for band-limited asynchronous DS-CDMA communications," IEEE Trans. Inform. Theory, vol. 48, pp. 1172-1185, May 2002.

[6] Y. C. Yoon, "A Simple and accurate method of probability of bit error analysis for asynchronous band-limited DS-CDMA systems," IEEE Trans. Commun., vol. 50, pp. 656-663, Apr. 2002.

[7] L.-L. Yang and L. Hanzo, "Performance of generalized multicarrier DSCDMA using various chip waveforms," IEEE Trans. Commun., vol. 51, pp. 748-752, May 2003.

[8] J. G. Proakis, Digital Communications, 3rd ed. Bershire, UK: McGraw Hill International Editions, 1995.

[9] N. C. Beaulieu and J. Cheng, "Precise error-rate analysis of bandwidthefficient BPS," vol. 52, pp. 149-158, Jan. 2004.

[10] G. P. Efthymoglou, V. A. Aalo, and H. Helmken, "Performance analysis of coherent DS-CDMA systems in a Nakagami fading channel with arbitrary parameters," IEEE Trans. Veh. Technol., vol. 46, pp. 289-297, May 1997.

[11] ARIB/Japan, Japan's Proposal for Candidate Radio Transmission Technology on IME-2000:W-CDMA, June 1998.

[12] N. Nakagami, "The $m$-Distribution, A General Formula for Intensity Distribution of Rapid Fading," in Statistical Methods in Radio Wave Propagation, W. G. Hoffman, ed. (Oxford, England: Pergamon, 1960).

[13] T. Eng and L. B. Milstein, "Coherent DS-CDMA performance in Nakagami multipath fading," IEEE Trans. Commun., vol. 43, pp. 11341143, Feb./Mar./Apr. 1995.

[14] V. Aalo, O. Ugweje, and R. Sudhakar, "Performance analysis of a DS/CDMA system with noncoherent $M$-ary orthogonal modulation in Nakagami fading," IEEE Trans. Veh. Technol., vol. 47, pp. 20-29, Feb. 1998.

[15] M.-S. Alouini and A. J. Goldsmith, "A unified approach for calculating error rates of linearly modulated signals over generalized fading channels," IEEE Trans. Commun., vol. 47, pp. 1324-1334, Sep. 1999.

[16] J. H. Cho and J. S. Lehnert, "Performance of a spatio-temporal matched filter for DS/SSMA communications with random quadriphase spreading sequences," IEEE J. Select. Areas Commun., vol. 18, pp. 1505-1515, May 2000.

[17] M. K. Simon and M.-S. Alouini, "A unified approach to the probability of error for noncoherent and differentially coherent modulation over generalized fading channels," IEEE Trans. Commun., vol. 46, pp. 16251638, Dec. 1998.

[18] M. K. Simon and M.-S. Alouini, "A unified approach to the performance analysis of digital communication over generalized fading channels," in Proc. IEEE, vol. 86, pp. 1860-1877, Sep. 1998.

[19] L. Hanzo, W. Webb, and T. Keller, Single- and Multi-carrier Quadrature Amplitude Modulation. New York: John Wiley \& Sons, Ltd, 2000. 